\title{
Characterization and Use of Wide-Field Fluorescence Microscopy and Image Restoration in Quantitative Live Cell Imaging
}

\author{
Melpomeni Platani*, Angus I Lamond*, and Jason R. Swedlow * \\ *Division of Gene Regulation and Expression, MSI/WTB Complex, University of Dundee, Dundee DD1 \\ 5EH Scotland
}

Digital fluorescence microscopy is now a standard tool for determining the localization of cellular components in fixed and living cells. Two fundamentally different imaging technologies are available for imaging fluorescently labelled cells and tissues, in either the fixed or living state. The laser scanning microscope uses a diffraction-limited focused beam to scan the sample and develop an image point by point. In addition, a pinhole placed in a plane confocal to the specimen prevents emitted out-of focus fluorescence from reaching the photomultiplier tube (PMT) detector. By combining spot illumination and selection of in-focus fluorescence signal, the laser scanning confocal microscope (LSCM) creates an image of the specimen largely free of out-of-focus blur [1]. By contrast, a wide-field microscope (WFM) illuminates the whole specimen simultaneously and detects the signal with a spatial array of point detectors, usually a charge-coupled device camera (CCD) [2]. This approach collects an image of all points of the specimen simultaneously and includes all the out-of-focus blurred light. Subsequent restoration by iterative deconvolution generates an estimate of the specimen, largely free of out-of-focus blur [3]. While many other fluorescence imaging modalities exist, these two methods represent the majority of the fluorescence imaging systems currently in use in biomedical research.

We (JRS) have recently completed a quantitative comparison of LSCM and WFM [4], focussing on commercially available versions of these microscopes. This analysis revealed that the most important parameter for comparing the performance of the two systems was the signal-to-noise ratio (SNR). Using both live cells and fluorescent beads standards, we showed that the intrinsic noise levels in commercial LSCMs are higher than in WFM, largely due to illumination power fluctuations. For low contrast specimens, especially live ones where illumination levels are kept low to preserve cell health, WFM gives superior performance. However, WFM did not perform well when thick tissues were examined. Again, the SNR dictated the choice. In thick tissue, out of focus noise in the WFM subsumes the in-focus signal, removing all contrast. For these specimens, the LSCM has a higher SNR and performs better. We are currently undertaking an analysis of a spinning disk microscope to assess how this system performs.

Based on this information, we have used a WFM to study the dynamics of the Cajal bodies in living cells. Cajal bodies (also known as coiled bodies), are subnuclear organelles that contain specific nuclear antigens, including small nuclear ribonuclear proteins (snRNPs) and a subset of nucleolar proteins $[5,6]$. Cajal bodies are localised in the nucleoplasm and are often found at the periphery of the nucleolus. We have constructed a stable HeLa cell line, HeLa ${ }^{\text {GFP-coilin }}$, that expresses the Cajal body marker protein, p80 coilin, fused to GFP [7]. The localisation pattern and biochemical properties of the GFP-coilin fusion protein are identical to the endogenous p80 coilin. Time lapse recordings on 63 nuclei of HeLa ${ }^{\text {GFP-coilin }}$ cells showed that all Cajal bodies move within the nucleoplasm. Movements included translocations through the nucleoplasm, joining of bodies to form larger structures and separation of smaller bodies from larger Cajal bodies. The GFP-coilin protein is dynamically associated with Cajal bodies as shown by changes in their fluorescence intensity over time. 
To determine the mechanism of Cajal body mobility, we tracked the trajectories of $350 \mathrm{CBs}$ in the nuclei of 50 living cells and calculated mean square displacements (MSD) of individual CBs [8]. Brownian diffusion is the primary mode of CB motility, although we also noted some examples of diffusion with flow. Plots of MSD vs $\square \mathrm{t}$ revealed that many CBs diffuse within a confined volume. To identify the nature of this confinement, we transfected $\mathrm{HeLa}^{\text {GFP-coilin }}$ cells with a plasmid encoding YFP-histone H2B and recorded the dynamics of $\mathrm{CBs}$ and chromatin by time-lapse fluorescence imaging. CBs that diffused within confined volumes were either immediately next to chromatin or surrounded by chromatin, suggesting that $\mathrm{CBs}$ can either be tethered to or confined by chromatin. Furthermore, calculation of the diffusion constant (D) of individual CBs through a time-lapse sequence showed large increases in D when chromatin constraints were relieved. We conclude that a single $\mathrm{CB}$ can switch between dynamic states, apparently because of changes in the physical association of the $\mathrm{CB}$ with chromatin.

This type of mobility is similar to what has been described previously as anomalous subdiffusion [9]. Indeed, using statistical analyses, we were able to show that Cajal body mobility is described by anomalous subdiffusion. Most interestingly, treatments that decrease the association of CBs with chromatin like ATP depletion and transcriptional inhibition also decrease the amount of subdiffusion.

Our results show that WFM combined with image restoration can produce novel analysis of cellular dynamics. In our case, we have used the low SNR levels of the WFM and the improvement in contrast obtained by deconvolution to characterize the dynamics of the nucleus in vivo.

\section{References}

[1] S. Inoué., Handbook of Biological Confocal Microscopy, J. Pawley, Plenum Press, New York, 1995. [2] D. A. Agard, Y. Hiraoka, P. Shaw and J. W. Sedat., Methods Cell Biol. 30 (1989) 353-377.

[3] J. R. Swedlow, J. W. Sedat and D. A. Agard., Deconvolution of Images and Spectra, P. A. Jansson, Academic Press, New York, 1997.

[4] J. R. Swedlow, K. Hu, P. D. Andrews, D. S. Roos and J. M. Murray., Proc. Soc. Natl. Acad. USA. in press (2002)

[5] A. G. Matera., Trends Cell Biol 9 (8) (1999) 302-309.

[6] J. G. Gall., Annu Rev Cell Dev Biol 16 (2000) 273-300.

[7] M. Platani, I. Goldberg, J. R. Swedlow and A. I. Lamond., J. Cell. Biol. 151 (2000) 1561-1574.

[8] M. Platani, A. I. Lamond and J. R. Swedlow., Submitted

[9] M. J. Saxton., Biophys J 70 (3) (1996) 1250-62.

[10] J.R.S. is a Wellcome Trust Career Development Fellow (054333). 